\title{
Polarization changes in solar radio emission caused by scattering from high-frequency plasma turbulence ${ }^{\star}$
}

\author{
V. G. Ledenev ${ }^{1}$, V. V. Tirsky ${ }^{2}$, V. M. Tomozov ${ }^{1}$, and P. Zlobec ${ }^{3}$ \\ ${ }^{1}$ Institute of Solar-Terrestrial Physics, Irkutsk, 664033, PO Box 4026, Russia \\ 2 Institute of Laser Physics, Irkutsk Branch, Russia \\ 3 Trieste Astronomical Observatory, via G. B. Tiepolo 11, 34131 Trieste, Italy
}

Received 29 March 2002 / Accepted 13 May 2002

\begin{abstract}
This paper deals with the scattering of electromagnetic radiation during propagation through a plasma layer with developed Langmuir turbulence. The ordinary component is slightly lowered, while the extraordinary component undergoes the most effective scattering. This leads to a change in the polarization characteristics of the original radiation, namely: the extraordinarily polarized emission can undergo a substantial decrease and even the polarization sign can be changed. As a consequence the radiation increases its polarization degree in the ordinary mode.

We performed calculations of the polarization of the radio emission propagating through a layer of turbulent plasma and examined the complex event that occurred on July 14, 2000; specifically, this event showed long-lasting emissions and the polarization varied both in time and in frequency range. Assuming that the variation of the polarization degree during the lifetime of the phenomenon is determined by the scattering from Langmuir turbulence, we obtained an estimate of the level of turbulence and of the magnetic field intensity in the emission region.
\end{abstract}

Key words. Sun: activity; flares; particle emission; radio radiation

\section{Introduction}

Studies of different types of evolution of complex type IV solar radio events and their polarization changes have been carried on over forty years (see, for example, Komesaroff 1958; Ruzdjak et al. 1986). Theories regarding polarization of solar radio events have been reported in many papers (e.g., Melrose \& Sy 1972; Dulk et al. 1976; Zlotnik 1981). Generally, at frequencies up to about $1 \mathrm{GHz}$, it was assumed that the emission is generated by a plasma mechanism, i.e. as a result of the conversion of longitudinal plasma waves (Langmuir turbulence) into electromagnetic waves (Ginzburg \& Zheleznyakov 1958). However, the quoted papers neglect the fact that the electromagnetic radiation should propagate through regions where high-frequency (Langmuir) turbulence is present. If the level of such turbulence is sufficiently high and the region it occupies is sufficiently extended, then the radio emission will be effectively scattered; part of the spectrum is shifted by a quantity that is equal to the electron plasma frequency. In such a case, as shown by Tirsky et al. (2000), the extraordinary mode is scattered more effectively. This means that the extraordinary component decreases more than the ordinary component and

Send offprint requests to: V. G. Ledenev,

e-mail: leden@iszf.irk.ru

* Figure 3 is only available in electronic form at http://www. edpsciences.org therefore even the polarization sign can be inverted. This effect can be more clearly distinguished in broadband continuous emission, like in type IV events. Such bursts are also most probably associated with extended regions of increased plasma turbulence.

We performed calculations of the polarization of the radio emission propagating through a layer of turbulent plasma and examined the complex event that occurred on July 14, 2000; specifically, this event showed long-lasting emissions and the polarization varied both in time and in frequency range. Assuming that the variation of the polarization degree during the lifetime of the phenomenon is determined by the scattering from Langmuir turbulence, we obtained an estimate of the level of turbulence and of the magnetic field intensity in the emission region.

\section{Polarization characteristics of the emission}

\subsection{Equations describing the scattering process}

The equations describing the process of Raman scattering of radio emission by turbulent Langmuir pulsations in plasma permeated by a magnetic field were obtained by Tirsky \& Tomozov (1998). In this section we examine the case of isotropic Langmuir turbulence with a spectral function $W(\boldsymbol{k})$ that is inversely proportional to the square of the wave number of Langmuir plasmons (Tsytovich 1971); in doing 
this we assume that the following conditions are satisfied: $\left(\omega_{\mathrm{He}} / \omega\right)^{2},\left(\omega_{\mathrm{He}} / \omega^{\prime}\right)^{2}<<1,\left(\omega_{\mathrm{pe}} / \omega\right)^{4},\left(\omega_{\mathrm{pe}} / \omega^{\prime}\right)^{4}<<1$,

$$
\int \frac{\mathrm{d} \bar{k}}{(2 \pi)^{3}} W(\boldsymbol{k})=W
$$

Here $\omega_{\text {pe }}$ and $\omega_{\mathrm{He}}$ are the Langmuir frequency and the gyrofrequency of electrons respectively, $\omega$ and $\omega^{\prime}$ are the frequencies of scattering and of scattered electromagnetic waves respectively; $\mathrm{W}$ is the energy of the Langmuir turbulence per unit of plasma volume. When scattering is taken into account, the equations describing the transport of radio emission in plasma with Langmuir turbulence in a homogeneous magnetic field (the optical thickness during scattering process is assumed of the order of one), provided that $\Delta \omega<\omega_{\mathrm{pe}}$ (where $\Delta \omega$ represents the width of the emission band), can be written as

$$
\begin{aligned}
\frac{\mathrm{d} I_{\omega}}{\mathrm{d} t} & =-\sigma_{0} f_{1}(\omega) I_{\omega}-\sigma_{0} f_{2}(\omega) V_{\omega} \cos \vartheta+v_{\mathrm{g}} S_{I}(\omega), \\
\frac{\mathrm{d} V_{\omega}}{\mathrm{d} t} & =-\sigma_{0} f_{1}(\omega) V_{\omega}-\sigma_{0} f_{2}(\omega) I_{\omega} \cos \vartheta+v_{\mathrm{g}} S_{V}(\omega) \\
\frac{\mathrm{d} U_{\omega}}{\mathrm{d} t} & =-\sigma_{0} f_{1}(\omega) U_{\omega}+v_{\mathrm{g}} S_{U}(\omega) \\
\frac{\mathrm{d} Q_{\omega}}{\mathrm{d} t} & =-\sigma_{0} f_{1}(\omega) Q_{\omega}+v_{\mathrm{g}} S_{Q}(\omega)
\end{aligned}
$$

Here $\vartheta$ is the angle between the axis collinear to the magnetic field vector and the direction of wave propagation $(\vartheta$ changes from 0 to $\pi / 2$ ), $I_{\omega}, U_{\omega}, Q_{\omega}$, and $V_{\omega}$ are the Stokes parameters; the functions $f_{1}, f_{2}$ and $\sigma_{0}$ are in the form

$$
\begin{aligned}
& f_{1}(\omega)=\left(\left(\left(\omega-\omega_{\mathrm{pe}}\right)^{2}-\omega_{\mathrm{pe}}^{2}\right)^{1 / 2}+\left(\left(\omega+\omega_{\mathrm{pe}}\right)^{2}-\omega_{\mathrm{pe}}^{2}\right)^{1 / 2}\right) / 2 \omega, \\
& f_{2}(\omega)=\left(\left(\omega_{\mathrm{He}}\left(\omega+\omega_{\mathrm{pe}}\right)^{-1}+\frac{\omega_{\mathrm{He}}}{\omega}\right)\left(\left(\omega+\omega_{\mathrm{pe}}\right)^{2}-\omega_{\mathrm{pe}}^{2}\right)^{1 / 2}\right. \\
& \left.+\left(\omega_{\mathrm{He}}\left(\omega-\omega_{\mathrm{pe}}\right)^{-1}+\frac{\omega_{\mathrm{He}}}{\omega}\right)\left(\left(\omega-\omega_{\mathrm{pe}}\right)^{2}-\omega_{\mathrm{pe}}^{2}\right)^{1 / 2}\right) / 2 \omega, \\
& \sigma_{0}=\frac{\pi}{3} \frac{\omega_{\mathrm{pe}}^{2}}{v_{\mathrm{g}}} \frac{T_{\mathrm{e}}}{m_{\mathrm{e}} c^{2}} \frac{W}{n_{\mathrm{e}} T_{\mathrm{e}}} \frac{1}{k_{1 \max }-k_{1 \min }} .
\end{aligned}
$$

Here $T_{\mathrm{e}}$ (in $\mathrm{J}$ ) is the electron temperature, $n_{\mathrm{e}}$ the plasma electron density, $c$ the speed of light in vacuum, $v_{\mathrm{g}}$ the group velocity of electromagnetic waves, and $k_{1 \max }$ and $k_{1 \min }$ are the maximum and minimum wave numbers of the Langmuir turbulence spectrum, respectively. For the quantities reported here (with the exception of temperature) we use the SI system. The functions $S_{I}, S_{U}, S_{Q}$ and $S_{V}$ describe the characteristics of the radio emission source in plasma.

\subsection{Change of the radio emission polarization as consequence of scattering in a turbulent plasma layer}

In the case where highly directive $(\Delta \theta<<\pi)$ radio emission propagates through a plane-parallel layer of turbulent plasma and the layer itself does not contain sources of radio emission of the frequencies under consideration $\left(S_{I}, S_{U}, S_{Q}\right.$, and $S_{V}=0$ ), from Eqs. (2)-(5) we obtain - for the intensity of the ordinary waves $I_{\mathrm{O}}=(I-V) / 2$ and the extraordinary waves $I_{\mathrm{X}}=(I+V) / 2$ after propagation through the layer - the following expressions:

$$
\begin{aligned}
& I_{\mathrm{X}}(z)=I_{\mathrm{X} 0} \exp \left(-\sigma_{0} z v_{\mathrm{g}}^{-1}(\cos \vartheta)^{-1}\left(f_{1}(\omega)+f_{2}(\omega) \cos \vartheta\right)\right) \\
& I_{\mathrm{O}}(z)=I_{\mathrm{O} 0} \exp \left(-\sigma_{0} z v_{\mathrm{g}}^{-1}(\cos \vartheta)^{-1}\left(f_{1}(\omega)-f_{2}(\omega) \cos \vartheta\right)\right)
\end{aligned}
$$

Here $\vartheta$ is the angle between the magnetic field vector and the direction of wave propagation ( $\vartheta$ changes from 0 to $\pi$ ), $I_{O O}$ and $I_{\mathrm{X} 0}$ are the intensities of the ordinary and of the extraordinary waves incident on the layer.

Figures 1a-c shows (when $\omega_{\mathrm{He}} / \omega_{\mathrm{pe}}=0.1,0.2,0.3$ ) $I_{\mathrm{X}}(z) / I_{\mathrm{O}}(z)$ versus optical thickness $\left(\tau=\sigma_{0} f_{1}(\omega) z / v_{\mathrm{g}}\right)$ of the layer for different $I_{\mathrm{X} 0} / I_{\mathrm{O} 0}$ when the emission is at the second harmonic of the plasma frequency. It is evident from the plots that, for the optical thickness of the order of one, the degree of polarization can change by $20-30 \%$. This is due to the more intense scattering of the extraordinary waves compared to the ordinary waves. With an increase in frequency, the ratio $I_{\mathrm{X}} / I_{\mathrm{O}}$, with constant optical thickness increases in almost the same way as it does with decreasing $\omega_{\mathrm{He}}$.

\section{Observations}

Figure 2 shows the dynamic spectrum of the July 14, 2000 event observed with the Phoenix ETH Zuerich spectrograph (Switzerland). From 10:27 to 10:40 UT there was intense broadband emission with a drift toward low frequencies. In Fig. 3 the profiles of the event are reported for six separate frequencies in total flux density and percentage of the circular polarization as observed with the radio-polarimeters of the INAF - Trieste Astronomical Observatory (Italy). At $237 \mathrm{MHz}$ before the beginning of the type IV event (at about 10:11), the left (L) circular polarization was given by the preexisting sources; one can see them in the Nancay radioheliograph pictures (picture rate $10 \mathrm{~s}$ ) (address http://www.obs-nancay.fr/html an/a rh.htm). Afterwards the number and the complexity of the radio sources increased; a few of them lasted less than 1 min, others remained longer. From about 10:33 up to the last picture (at 10:45) shown in the Nancay data, only one source was present and during that time the strongest burst (maximum about $8000 \mathrm{sfu}$ ) evolved slowly. It is remarkable that during its lifetime the polarization changed from zero to $50 \%$ in the right (R) circular sense, the polarization and the burst profile variation being completely different in shape. That "out of phase" trend can be explained by the theory proposed here, when the ordinary mode was R. At $327 \mathrm{MHz}$ during the initial phase of the event the polarization was slightly L. During that time the sources represented by the Nancay radioheliograph were generally complex in shape and number. The "great" burst lasting from 10:27 up to 10:40 was so strong that for about $3 \mathrm{~min}$ the receiver was saturated (the saturation level corresponds to 

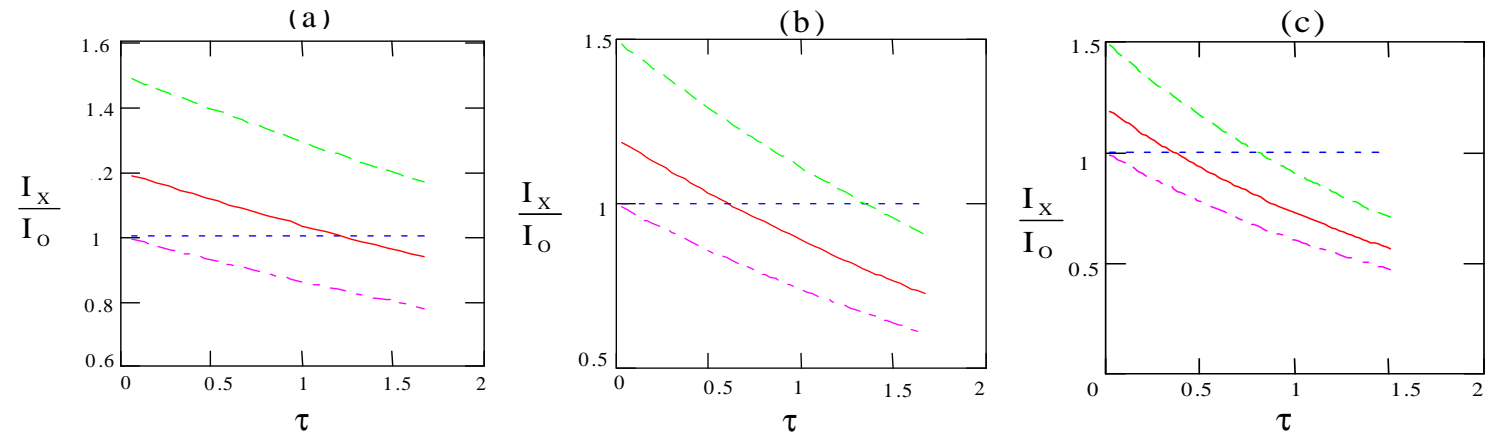

Fig. 1. Dependence of the intensity ratio of ordinary and extraordinary waves at the second harmonic of the plasma frequency on the optical thickness for different initial intensity ratios and for $\omega_{\mathrm{He}} / \omega_{\mathrm{pe}}=0.1 \mathbf{a}$ ), for $\omega_{\mathrm{He}} / \omega_{\mathrm{pe}}=0.2 \mathbf{b}$ ), for $\omega_{\mathrm{He}} / \omega_{\mathrm{pe}}=0.3 \mathbf{c}$ ); propagation along the magnetic field $(\alpha=0)$.

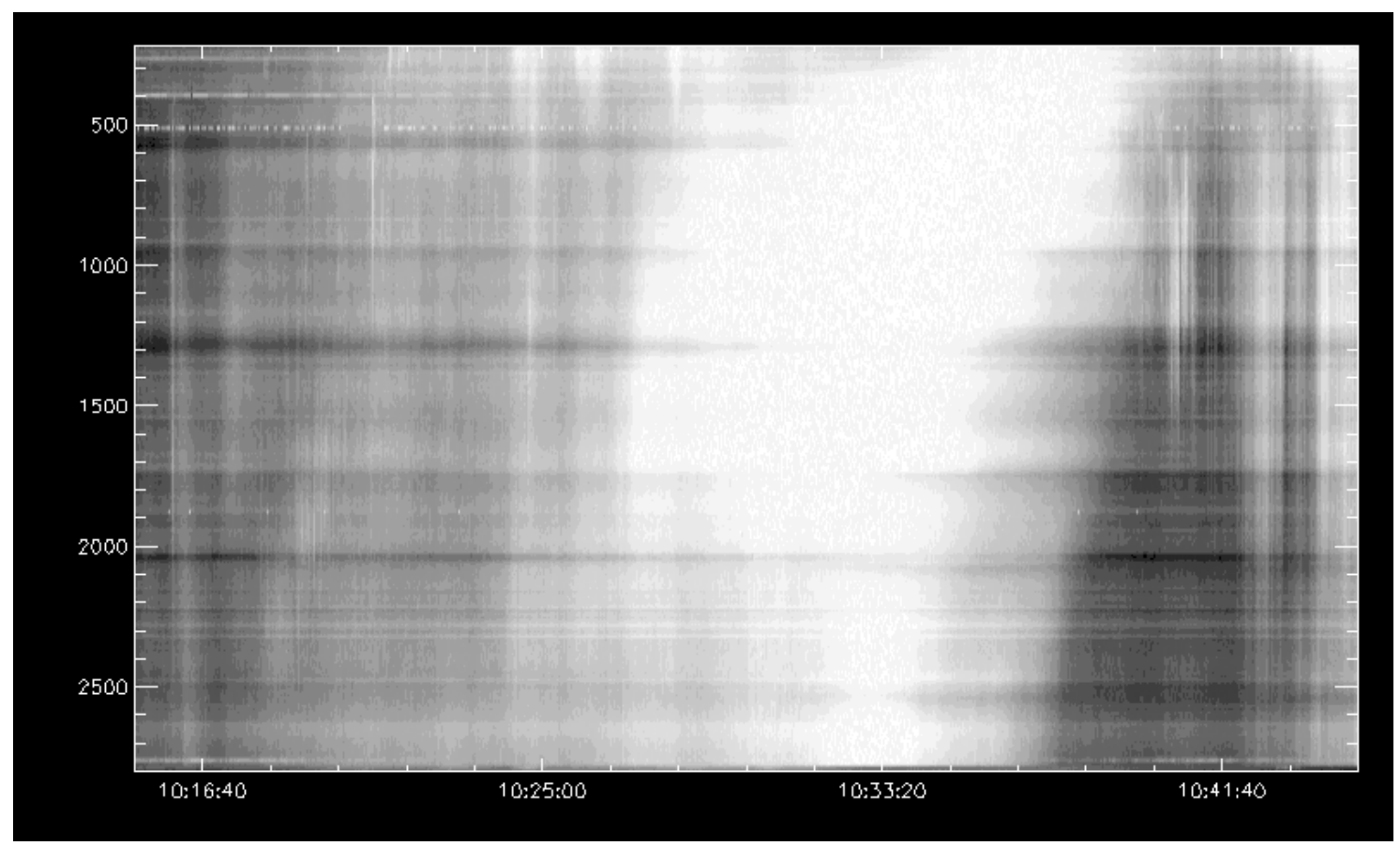

Fig. 2. The radio spectrum of the 14 July 2000 event observed with the Phoenix ETH Zuerich spectrograph. The vertical axis plots the frequency in $\mathrm{MHz}$, the horizontal axis plots the time (UT).

about $10000 \mathrm{sfu}$ ); just after the missing part, at 10:34, the polarization passed from zero to $70 \mathrm{R}$ in $4 \mathrm{~min}$, in that occasion also its trend was not "in phase" with the decaying part of the burst intensity, even if the emitting source (from 10:33 to $10: 45)$ was unique.

At $408 \mathrm{MHz}$ practically all the enhancements were R polarized (no Nancay radioheliographic data at that and at higher frequencies). Analogously, as at lower frequencies, the trend of the "great" burst (at this frequency it was also evolving rather slowly (peak at about 10:32)) was "out of phase" with respect to the time evolution of the polarization. In particular, it appears that the $\mathrm{R}$ polarization enhancement (up to 60\%) was "delayed" in comparison to the time evolution of the "great" burst intensity.

At $610 \mathrm{MHz}$ the pre-existing background was strongly L; it was probably generated by a low interference signal. The type IV event started at about 10:13 and its polarization decreased up to 10:31. At that time an abrupt increase of the flux accompanied the change of the polarization sign and the $\mathrm{R}$ polarization increased up to $50 \%$; it is important to mention that also at that frequency the evolution of the polarization was "delayed" with respect to the "great" burst evolution (maximum at 10:33). From 10:38 to $10: 42$ practically only the low continuum was present, therefore it is not surprising that the polarization percentage was similar to the value present at the initial phase of the type IV event. The last enhancement that started at 10:43 was also R polarized. At $1420 \mathrm{MHz}$ the event started at about 10:12. It was slightly $\mathrm{L}$ polarized for about $10 \mathrm{~min}$, afterwards all its components showed opposite polarization (R) with different percentage values. Meanwhile the slowly decaying continuum from 10:33 to 10:49 was generally slightly L. The "great" burst with maximum at 10:31 was not so prominent as at lower frequencies and its trend and polarization evolution appear rather "in phase", as normally happens for strongly polarized bursts emitted by a plasma mechanism. The strongest component happened rather late, between 10:50 and 10:55, and 
in that case the polarization trend (maximum of polarization (65 R) at 10:54) was still "delayed" in comparison to the burst evolution that showed its maximum at 10:51.

At the highest frequency $(2695 \mathrm{MHz})$ the type IV event showed a rather different aspect. It started earlier (at 10:05) and the intensity profile and the polarization trend were completely different than at lower frequencies. The background before and after the event was about $3 \mathrm{~L}$, during the event it was 4-12 L, with a mean value of about $8 \mathrm{~L}$. It may be quite safely suggested that the event was generated by gyrosynchrotron mechanism. The source was probably present at the top of a magnetic loop or of an arch system, i.e. in the neighborhood of the source the emission was propagating across the magnetic field. Since the associated flare was near the center of the solar disk, it is plausible that the emission was weakly polarized (Dulk \& Marsh 1982). The type IV event recorded on July 14, 2000 changed drastically its polarization (and generally also the change of sign was present) during the strongest slowly evolving burst (Figs. 3a-e) and its intensity evolution was out "of phase" with respect to the polarization trend. (At $2695 \mathrm{MHz}$ (Fig. 3f) it is most probable that the emission mechanism was different.) Such a phenomenon was generated in a sufficiently extended region of the solar corona (for instance in a coronal arch, see Stepanov 1973) where a high level of plasma (Langmuir) turbulence was excited. When propagating through such a region the radio waves were scattered and their polarization characteristics were changed according to the proposed theory.

Solar images recorded by the Nancay radioheliograph show the emission positions at 164, 237 and $327 \mathrm{MHz}$ before and after the presence of the "great" burst with slow evolution at $237 \mathrm{MHz}$ (Fig. 4). Since during its lifetime the source position was not moving, the polarization should maintain its value; on the contrary we recorded a strong variation in the polarization and that trend was "out of phase" with respect to the burst evolution. It is natural to relate such a phenomenon to the passage of the electromagnetic waves through the highly turbulent layer. In such a layer, during the growth phase of the burst the turbulence level increases together with the optical thickness and also the dimensions grow. The growth of the size is also related to the slow frequency drift of the low frequency boundary of the "great" burst (Fig. 2). Since subsequently the polarization degree increased (in the $\mathrm{R}$ sense) during the burst lifetime, the $\mathrm{R}$ polarization of the emission must correspond to the ordinary mode. There are also other mechanisms that explain the polarization reversal. They include primarily the reversal of the sense of rotation of the electric field vector during the propagation through a region of a quasi-transverse magnetic field (Zheleznyakov 1970). In the event of July 14, 2000 however, this mechanism does not seem to work as the magnetic field structure along the propagation path should not change substantially, the source position being at nearly the same place. Such mechanisms can change the polarization sense but cannot explain the "out of phase" trend between intensity and polarization evolution. Consequently, the most probable mechanism for the increase of the polarization degree was the scattering of electromagnetic radiation due to strong enough plasma turbulence.

\section{Discussion}

It is well known (Zheleznyakov 1970; Solar Radiophysics 1985) that at frequencies below $1 \mathrm{GHz}$ the plasma emission mechanism is regarded as the highly predominant one. It is further assumed that sufficiently intense Langmuir turbulence is excited in the emitting region. The calculations presented above show that the extraordinarily polarized emission is scattered more effectively than the ordinarily polarized one. When highly directed extraordinarily polarized electromagnetic radiation crosses a region of dense enough Langmuir turbulence, such an emission is scattered. Meanwhile, for the ordinarily polarized radiation the effect is substantially weaker. Therefore the polarization degree will be modified, i.e. the percentage of the ordinary mode will increase. The formation time of sufficiently intense Langmuir turbulence introduces a time shift to make the polarization profile delayed with respect to the evolution of the "great" burst of a rather long duration. In that way it is possible to explain the polarization trend of the most prominent bursts during the July 14, 2000 event at frequencies of 237, 327, 408, 610 and $1420 \mathrm{MHz}$.

The increase of the polarization percentage during the lifetime of a burst of slow evolution that is caused by scattering of emission from Langmuir turbulence allows us to estimate the level of Langmuir turbulence and the magnetic field in the emitting region. In our calculations we assumed that the emission is contained in a narrow angle. Actually, the directivity pattern of the radiation occupies a finite interval of angles (Zheleznyakov 1970; Kundu 1965). However, for the plasma mechanisms of emission, the condition $\Delta \theta<\pi$ is usually satisfied (Zheleznyakov \& Zaitsev 1970; Ledenev 1994) and hence the results from the above calculations can be used to estimate the scattering effectiveness. Assuming that the optical thickness for the scattering process $\tau=\sigma_{0} f_{1}(\omega) z / v_{g}$ is of the order of one, from formulas (6) and (8) we obtain the following estimated energy of Langmuir plasmons for $\omega \approx 2 \omega_{\mathrm{pe}}$ :

$W=3 n_{\mathrm{e}} m_{\mathrm{e}} c^{3} / \pi \omega_{\mathrm{pe}} L \approx 1.7 \times 10^{-5} n_{\mathrm{e}}^{1 / 2} m_{\mathrm{e}} c^{3} / L$,

where $n_{\mathrm{e}}$ is the electron density, $m_{\mathrm{e}}$ the mass of an electron, $c$ the velocity of light, $\omega_{\text {pe }}$ the electron plasma frequency and $\mathrm{L}$ the size of the region occupied by turbulence, respectively. When $L \sim 10^{7} \mathrm{~m}$ and $n_{\mathrm{e}} \sim 10^{14} \mathrm{~m}^{-3}$ we obtain $W \sim 10^{-7} \mathrm{~J} / \mathrm{cm}^{3}$ or $10^{-6} \mathrm{erg} / \mathrm{cm}^{3}$.

By examining the propagation of the radio waves through a plane-parallel layer of turbulent plasma, we can estimate the degree of polarization of emission. From formulas (9) and (10) we have:

$$
\begin{aligned}
& \eta=\frac{I_{\mathrm{X}}(z)-I_{\mathrm{O}}(z)}{I_{\mathrm{X}}(z)+I_{\mathrm{O}}(z)} \\
& =\frac{I_{\mathrm{X} 0}\left(f_{1}(\omega)+f_{2}(\omega) \cos \vartheta\right)-I_{\mathrm{O} 0}\left(f_{1}(\omega)-f_{2}(\omega) \cos \vartheta\right)}{I_{\mathrm{X} 0}\left(f_{1}(\omega)+f_{2}(\omega) \cos \vartheta\right)+I_{\mathrm{O} 0}\left(f_{1}(\omega)-f_{2}(\omega) \cos \vartheta\right)} .
\end{aligned}
$$

If the incident emission is unpolarized, then

$\eta=\frac{f_{2}(\omega) \cos \vartheta}{f_{1}(\omega)}$

When $\omega \approx 2 \omega_{\mathrm{pe}}$ we have $f_{1}(\omega) \approx \sqrt{ } 2 \omega_{\mathrm{pe}}$ and $f_{2}(\omega) \approx$ $(5 \sqrt{ } 2 / 3) \omega_{\mathrm{He}}$. Consequently, $\eta=(5 / 3)\left(\omega_{\mathrm{He}} / \omega_{\mathrm{pe}}\right) \cos \vartheta$. This 

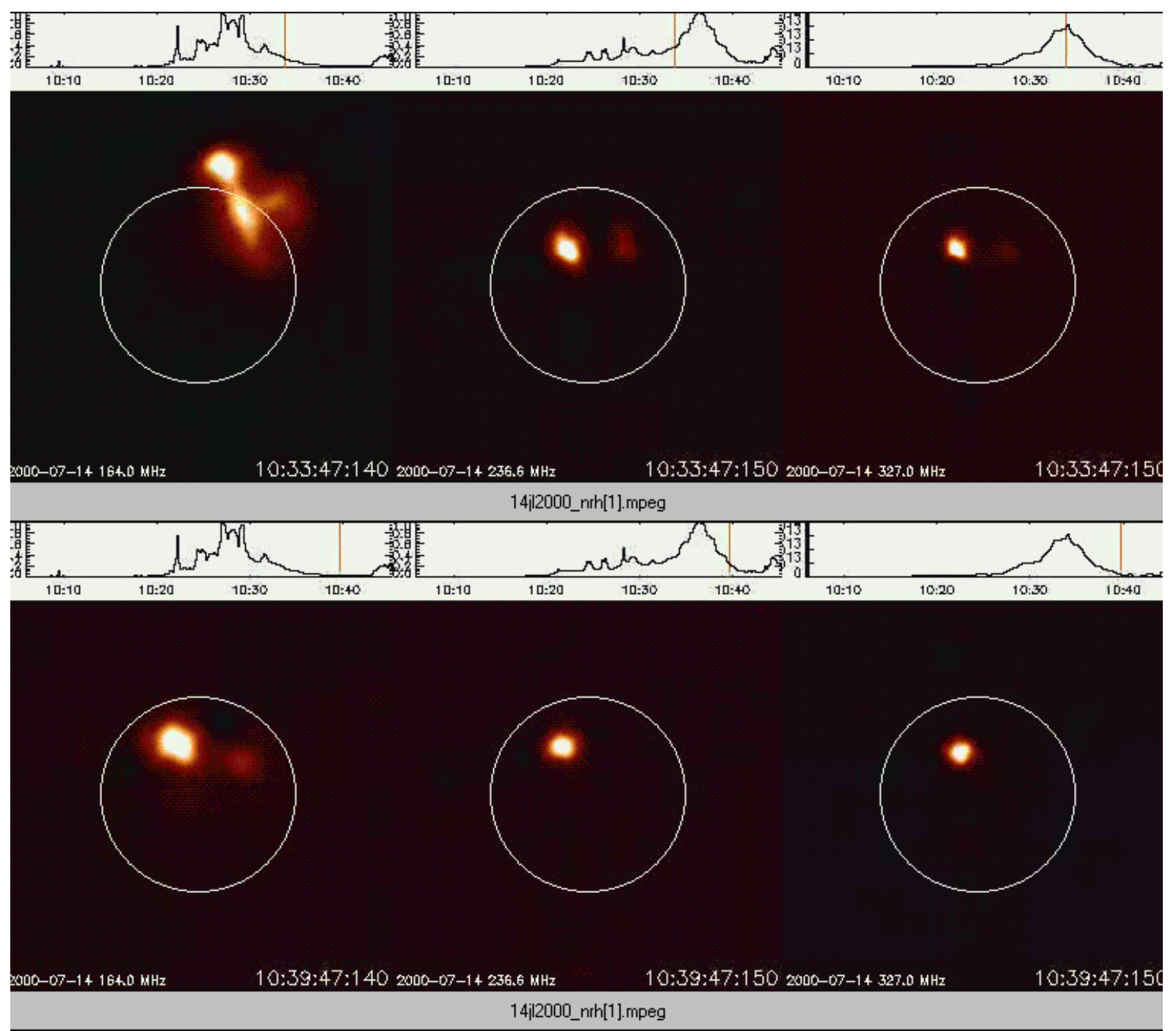

Fig. 4. Solar images obtained by the Nancay radioheliograph at frequencies 164, 237 and $327 \mathrm{MHz}$ prior to (up) and after (down) the polarization reversal at $237 \mathrm{MHz}$.

gives an estimate of the ratio $\omega_{\mathrm{He}} / \omega_{\mathrm{pe}}$ and of the magnetic field. For the event of July 14, 2000, namely for a polarization degree of $50 \%$ we find $\omega_{\mathrm{He}} / \omega_{\mathrm{pe}} \sim 0.3$ and for $\omega \sim 10^{9} \mathrm{~s}^{-1}$ the magnetic field $B \sim 10 \mathrm{G}$, provided that the emission is generated at the harmonic of the plasma frequency.

\section{Conclusion}

When radio waves propagate through a region of plasma with strongly developed Langmuir turbulence, they undergo an intense scattering and part of the emission polarized in the extraordinary mode is shifted (in the spectrum) by a value equal to the electron plasma frequency. In this case the extraordinary waves are more scattered than the ordinary ones. This leads to a decrease of the original emission in the extraordinary mode by $20-30 \%$ when the optical thickness of the scattering process is near one and can even lead to polarization reversal. The decrement of the ordinarily polarized component is smaller, therefore the resulting polarization increases in the sense of the ordinary mode. This effect is most clearly visible in long-lasting broadband bursts.

We apply the proposed formulas by considering as an example the strongest slowly evolving burst that appeared during the July 14, 2000 type IV event. During the lifetime of such a burst the polarization increased from about zero to $50 \%$, but such variations were not in phase with the burst intensity trend. Such a behaviour indicates that the original emission is scattered, i.e. the percentage of polarization in the ordinary mode increases as the optical thickness grows, as a consequence of the increase of the level of Langmuir turbulence during the growth phase of the burst and the subsequent increase of the turbulent region size.

Acknowledgements. The authors acknowledge Dr Messmer of the ETH - Zuerich (for the dynamical spectrum), Dr Klein of the Meudon Observatory (for the images of the Nancay radioheliograph) and Dr Grechnev (for help in preparing the dynamical spectrum). They also wish to thank Mr Padovan (for preparing the programs for radio data acquisition and for data reduction), Mr Fornasari (for preparing the plots) and Mr Coretti (for his help to P.Z. in computer 
problems) of the INAF - Trieste Astronomical Observatory. The work was supported by the Russian Fundation of Basic Research (grants 00-15-96710 and 00-02-16819).

\section{References}

Dulk, G., \& Marsh, K. 1982, ApJ, 259, 350

Dulk, G., Smerd, S. F., Mc Queen, R. M., et al. 1976, Sol. Phys., 49, 369

Ginzburg, V. L, \& Zheleznyakov, V. V. 1958, AZh, 35, 694 (in Russian)

Komesaroff, M. 1958, Aust. J. Phys., 11, 201

Kundu, M. 1965, Solar Radioastronomy (New York, Interscience Publ.)

Ledenev, V. G. 1994, A\&A, 285, 1019
Melrose, D. B., \& Sy, W. N. 1972, Australian J. Phys., 25, 387

Ruzdjak, V., Vrsnak, B., Zlobec, P., \& Schroll, A. 1986, Sol. Phys., 104, 169

Solar Radiophysics 1985, ed. by D. J. McLean, \& N. R. Labrum (Cambridge University Press)

Stepanov, A. V. 1973, AZh, 50, 1243 (in Russian)

Tirsky, V. V., \& Tomozov, V. M. 1998, Izvestiya VUZ’ov, Fizika, 4, 102 (in Russian)

Tirsky, V. V., Ledenev, V. G., \& Tomozov, V. M. 2000, Phys. Scri., 62, 196

Tsytovich, V. N. 1971, Theory of Turbulent Plasma, Atomizdat Zheleznyakov, V. V. 1970, Radio Emission of the Sun and Planets (Pergamon Press)

Zheleznyakov, V. V., \& Zaitsev, V. V. 1970, AZh, 47, 60 (in Russian)

Zlotnik, E. Ya. 1981, A\&A, 101, 250 